\title{
Use of Antiserum Agar for Detection of Haemophilus influenzae Type b in the Pharynx
}

\author{
RICHARD H. MICHAELS ${ }^{(24)}$ AND FRANCES E. STONEBRAKER \\ Children's Hospital of Pittsburgh and the Department of Pediatrics, University of Pittsburgh School of Medicine, \\ Pitsburgh, Pennsylvania, USA \\ JOHN B. ROBBINS \\ Developmental Immunology Branch, National Institute of Child Health and Human Development, National \\ Institutes of Health, Bethesda, Maryland, USA
}

\section{Extract}

An antiserum agar medium was-evaluated to determine its usefulness and accuracy in the study of the prevalence of Haemophilus influenzae type $b(H I B)$ in the pharynx of infants and young children. The effects of varying the concentration of ingredients (Levinthal base, antiserum, and bacitracin) were demonstrated. The medium could be stored at $4^{\circ}$ for periods up to 12 weeks with no loss of effectiveness. All halo-producing organisms detected after 24-40 $\mathrm{hr}$ of incubation were found to be HIB. When fewer than 20 colonies of HIB were found on antiserum agar, HIB could not be detected on either rabbit blood agar or chocolate human blood agar simultaneously inoculated with throat swab fluids. Radial streaking with a $0.001-\mathrm{ml}$ calibrated loop yielded at least twice as many isolated colonies with halos than did inoculation by streaking from a swabbed area of the plate.

Oropharyngeal culture of 543 healthy Pittsburgh preschool children indicated that the prevalence of HIB is about $3 \%$ in children age 6 months through 3 years; HIB colonization was not observed during the first 6 months of life. There was a much higher carrier rate $(65 \%)$ ) for siblings of patients with HIB meningitis or epiglottitis, as well as a greater intensity of colonization; nearly three-quarters of the sibling carriers had over $5 \times 10^{4} \mathrm{HIB}$ colonies/throat swab.

The medium is dependent on large amounts of potent and highly specific antiserum, a concentrated Levinthal base, and a refined agar. Its advantages are rapidity, sensitivity, specificity, and a potential for quantitation. The medium should be useful for carrier rate studies and for investigations of the role of HIB in such conditions as pneumonia, sinusitis, conjunctivitis, and orbital cellulitis of childhood.

\section{Speculation}

The high intensity of pharyngeal colonization of siblings of children with HIB meningitis may be important in the pathogenesis of this disease. Recent work in an infant rat model (9) suggests that the development of HIB meningitis requires a large inoculum of organisms. Close contact of a susceptible child with someone intensely colonized with HIB could provide this large inoculum.

Haemophilus influenzae type $\mathrm{b}$ is the most common cause of endemic bacterial meningitis and is sometimes responsible for other serious illnesses in children, including epiglottitis and septic arthritis (14). Epidemiologic studies have suggested that HIB is present in the pharynx of only $0.5-3 \%$ of normal infants and children and is rarely found in adults (16). However, these estimates of frequency are based on relatively small numbers chiefly because of the time-consuming bacteriologic differentiation of HiB from other organisms in the pharynx, particularly the much more prevalent nonencapsulated $H$. influenzae, $H$. influenzae of other capsular types, Haemophilus parainfluenzae and Haemophilus hemolyticus. Recently, an antiserum agar technique originally described by Petrie (11) and later used by Pittman (12) and her associates and by Ouchterlony (10), was utilized by Bradshaw et al. (2) to study bacterial antigens cross-reactive with the capsular polysaccharide of HIB in children and in laboratory animals. In the present report, an antiserum agar medium was evaluated to determine its usefulness and accuracy in the study of the prevalence of HIB in infants and young children.

\section{MATERIAL AND METHODS}

This technique utilized hyperimmune HIB antiserum and bacitracin incorporated into clear agar with a Levinthal base. Identification was achieved by observing halos of precipitation surrounding individual colonies of HIB.

HIB antisera was prepared in goats, sheep, and burros as described previousiy (13). The concentration of precipitating anti-type b antibody ranged from $1.8-3.4 \mathrm{mg}$ protein $/ \mathrm{ml}$. Levinthal base with added NAD (17) at $1.0 \mu \mathrm{g} / \mathrm{ml}$ was stored at $-20^{\circ}$. Bacitracin (18), brain heart infusion (BHI) (19), and SeaKem agarose $(20)$ were used without modification.

Levinthal base was prepared by the rapid addition of $550 \mathrm{ml}$ freshly drawn sterile heparinized horse blood to 2 liters of $\mathrm{BHI}$ maintained at $90^{\circ}$ with stirring. The stirring was continued at room temperature for $10 \mathrm{~min}$. The supernatant fluid was decanted into sterile steel bottles (21) and centrifuged at $7,000 \mathrm{rpm}, 4^{\circ}$, for $20 \mathrm{~min}$. The supernatant fluid was poured into a 2 -liter flask with $0.5 \mathrm{~g}$ NAD in $20 \mathrm{ml}$ phosphate-buffered saline. The fluid was mixed and dispersed in sterile $100-\mathrm{ml}$ bottles and stored at $-20^{\circ}$.

BHI $(18.5 \mathrm{~g})$, agarose $(10 \mathrm{~g})$, and distilled water $(500 \mathrm{ml})$ were added to a 2-liter flask with a magnetic stirring bar, autoclaved, mixed to insure thorough blending of the agar, and equilibrated at $50^{\circ}$ in a waterbath. Levinthal base $(100 \mathrm{ml})$ and $\mathrm{HIB}$ antiserum $(40-45 \mathrm{ml})$, equilibrated in a waterbath at $50^{\circ}$, were added by carefully pouring down the flask wall, mixing gently, and adding bacitracin to a final concentration of 5 units $/ \mathrm{ml}$. The medium was poured (approximately $16-18 \mathrm{ml}$ into plastic petri dishes, $100 \times 15$ $\mathrm{mm}$ ), allowed to gel, and stored in sealed plastic bags at $4^{\circ}$ until use. Each batch was tested for adequate growth and halo formation with stock HIB strains.

Pharyngeal swabs were immersed in $1.0 \mathrm{ml}$ Trypticase soy 
broth (19) immediately after the specimen was obtained, and allowed to stand at room temperature for $1-4 \mathrm{hr}$ before inoculation of the platè. (Early experiments using Levinthal broth as transport medium showed no decline in HIB titer after overnight storage at $4^{\circ}$, but about a $50 \%$ loss using Trypticase soy broth. For the briefer holding times employed in these studies there was no decline with either medium.) At the time of inoculation the swab was squeezed out, removed, and the fluid mixed. A platinum loop calibrated to deliver $0.001 \mathrm{ml}$ was employed for radial streaking of the agar plate. The same plate was then also more heavily inoculated by running the swab around the extreme periphery; rarely the swabbed area revealed HIB colonies when none were found in the area of radial streaking.

Plates were incubated for $24-40 \mathrm{hr}$ before observation. An illuminator was used which allowed for oblique lighting with observation against a dark background. At $24 \mathrm{hr}$ the HIB colonies were iridescent but halos were usually faint. Isolated colonies at 40 hr were approximately $3-4 \mathrm{~mm}$ in diameter with a moist, light gray, translucent appearance. Halos extended about $2 \mathrm{~mm}$ out from the edge of the colony and were more distinct at the periphery. Figure 1 demonstrates pharyngeal cultures photographed approximately $40 \mathrm{hr}$ after inoculation. Colonies in the central portion of the plate $(0.001 \mathrm{ml}$ inoculum $)$ were counted to provide semiquantitative data.

\section{RESULTS}

EFFECT OF VARYING CONCENTRATION OF INGREDIENTS

Experiments indicated that Levinthal base had to be $1 / 6-1 / 8$ the total volume of the media. Agar made up with the $1 / 10$ volume Levinthal base produced small HIB colonies with indistinct halos. The amount of antiserum necessary for easily detectable halos varied with the concentration of precipitating antibody. In one experiment a $25 \%$ reduction or increase in antiserum concentration resulted in no appreciable difference in the appearance of halos, and no enhancement or inhibition of growth of HIB. In general, approximately $0.1 \mathrm{mg}$ antibody protein $/ \mathrm{ml}$ media was sufficient to produce distinct halos. The concentration of bacitracin could vary from 320 bacitracin units $/ \mathrm{ml}$ without reduction in the growth of $\mathrm{HIB}$, and 3 units $/ \mathrm{ml}$ appeared to be as effective in inhibiting other bacteria as the higher concentrations tested.

\section{EFFECTS OF STORAGE OF PLATES}

Plates could be stored at $4^{\circ}$ in sealed plastic bags for periods up to 12 weeks with no loss in effectiveness. At 18 weeks some desiccation of agar was noted, and inoculation of a stock strain of HIB at that time resulted in colonies and halos which were somewhat reduced in size as compared with those on more recently prepared plates.

\section{SPECIFICITY OF MEDIUM}

Initially, colonies with halos from throat swab specimens were tested by slide agglutination using type specific $H$. influenzae antiserum (22). They were also tested to determine Gram stain morphology, hemolytic potential on rabbit blood agar, and growth dependency on $\mathrm{X}$ and $\mathrm{V}$ factors. All halo-producing organisms detected at $24-40 \mathrm{hr}$ were HIB, consistent with previous results (13). Specifically, no cross-reacting Escherichia coli strains were found in the pharynx of any of the children tested. Further incubation (3-4 days) resulted in the appearance of halos around some strains of $H$. parainfluenzae. These colonies were easily recognized since they were smaller and drier and their halos were much less distinct than those surrounding HIB, particularly at the periphery. None of these $H$. parainfluenzae strains were agglutinated by HIB antiserum (22).
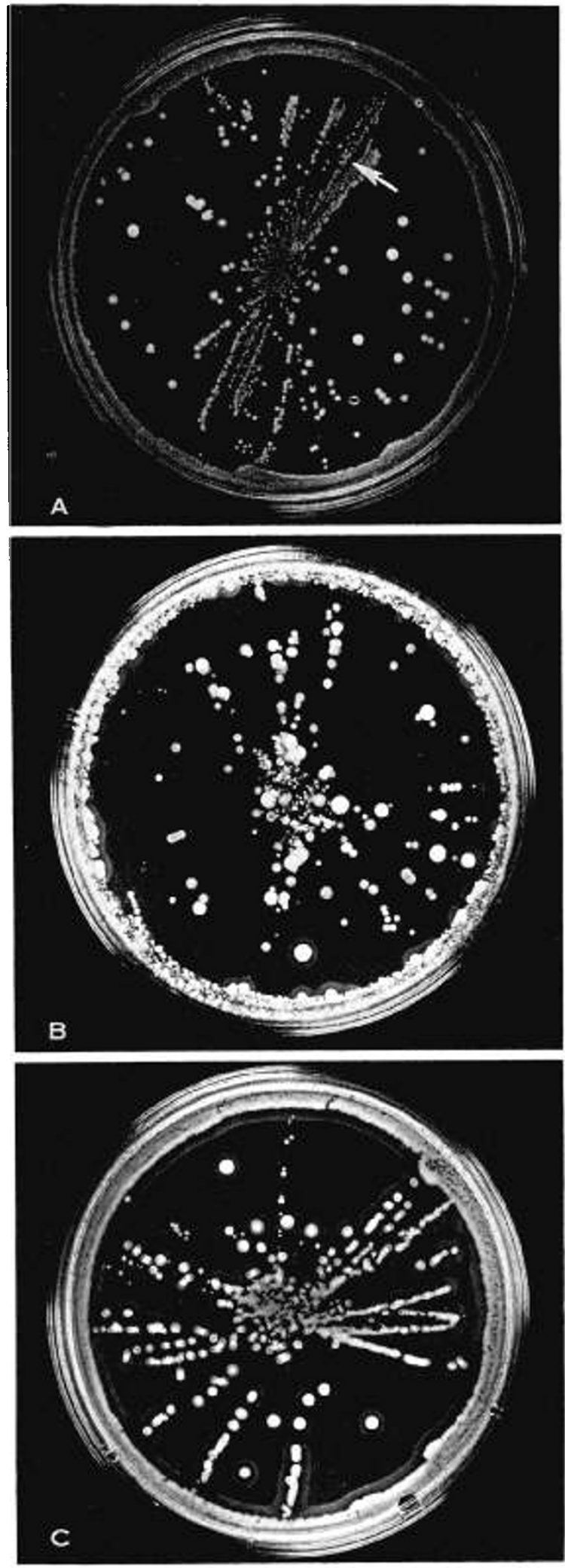

Fig. 1. Appearance of antiserum agar plates $40 \mathrm{hr}$ after inoculation with pharyngeal swab fluids from three children $(A, B$, and $C)$, showing differences in intensity of colonization with Haemophilus influenzae type B (HIB) (colonies with surrounding halos). Plate from A has one HIB colony whereas plate from $B$ has 10 and plate from $C$ has over 100 HIB colonies in central radially streaked areas. Heavily inoculated rim areas of these plates show no growth, moderate growth, and nearly confluent growth of HIB, respectively. 


\section{COMPARISON WITH OTHER MEDIA}

Agar plates with rabbit blood or chocolate human blood with Fildes supplement (19) are routinely used in the laboratories at the Children's Hosiital of Pittsburgh and elsewhere for the isolation of $H$. influenzae from spinal fluid and other specimens. Colony size was similar on chocolate and antiserum agar media; colonies were much smaller on rabbit blood agar. Quantitative testing showed that HIB could not be detected on either rabbit blood or chocolate agar plates when fewer than 20 colonies were found on antiserum agar plates simultaneously inoculated with throat swab fluids. Overgrowth of competing pharyngeal organisms and the time-consuming identification of suspected $H$. influenzae by standard procedures, including slide agglutination with type-specific antiserum, characterized isolation methods with conventional media.

Sheep blood agar plates were also tested since this medium is so commonly employed for the isolation of $\beta$ hemolytic streptococci. Sheep blood was even more unsatisfactory than rabbit blood and chocolate human blood for the detection of small numbers of HIB in throat swab fluids. The synthesis of $\mathrm{V}$ factor by pharyngeal staphylococci and other organisms apparently did not compensate for the inhibitors of $\mathrm{V}$ factor which are present in unheated sheep blood. In one experiment where appropriate amounts of HIB were added to throat swab fluids to yield 20-30 colonies/plate on antiserum agar medium, HIB could not be isolated from any of the 20 specimens simultaneously inoculated on sheep blood agar.

\section{COMPARISON OF PLATING TECHNIQUES}

A comparison of radial streaking using a calibrated loop (see Materials and Methods) with a commonly used method of inoculating throat cultures (platinum loop streaking from a section of the plate heavily inoculated by swab) was carried out with seven children known to be pharyngeal carriers of HIB. Antiserum agar plates which had been inoculated by radial streaking yielded at least twice as many isolated colonies with halos as did simultaneously inoculated antiserum agar plates utilizing the more common technique. Halo formation sometimes occurred around colonies buried among other organisms in the section of the plate which had been inoculated with the swab, but HIB colonies in the swabbed area can be obscured by other organisms. An additional advantage of the radial streaking technique is its potential for providing semiquantitative data.

\section{PREVALENCE OF HIB IN INFANTS AND YOUNG CHILDREN}

Throat swabs were collected from 543 healthy preschool children visiting six health department "well baby" clinics and the offices of eight pediatricians in different areas of metropolitan Pittsburgh. Children with colds and other respiratory illnesses were carefully excluded because of the reported higher frequency of pharyngeal HIB in association with these conditions (15). Oropharyngeal rather than nasopharyngeal swabs were collected because of the better acceptance by the children and because earlier studies had suggested that isolation rates are similar; rates for $H$. influenzae were actually found to be slightly higher when throat swabs rather than "pernasal" swabs were employed in older children and adults (8).

The results of our surveys of normal subjects are summarized in Table 1. The highest percentage of positive cultures (about $3 \%$ ) was found in children age 6 months through 3 years; HIB colonization was not observed during the first 6 months of life. There was a much higher carrier rate $(65 \%)$ for siblings of patients hospitalized with HIB meningitis or epiglottitis as well as a greater intensity of colonization in these individuals; nearly three-quarters of the sibling carriers had over 50 colonies/plate $\left(5 \times 10^{4}\right.$ HIB colonies/ swab). Similar observations on the frequency of HIB carriage in family contacts of diseased individuals have been reported (16), but we could find no reports of attempts to quantitate pharyngeal HIB in such children. Correlations between concentration of serum
Table 1. Frequency of detection of Haemophilus influenzae type $b$ $(H I B)$ in the oropharynx of healthy preschool children in Pittsburgh

\begin{tabular}{|c|c|c|c|c|c|}
\hline \multirow[b]{2}{*}{ Age } & \multirow{2}{*}{$\begin{array}{l}\text { Total No. } \\
\text { children } \\
\text { tested }\end{array}$} & \multicolumn{3}{|c|}{ No. colonies/plate } & \multirow{2}{*}{$\begin{array}{l}\text { Total No. } \\
\text { children } \\
\text { positive }(\%)\end{array}$} \\
\hline & & $1-10$ & $11-50$ & $>50$ & \\
\hline Birth -5 mo & 91 & 0 & 0 & 0 & $0(0)$ \\
\hline $6-11 \mathrm{mo}$ & 97 & 2 & 0 & 1 & $3(3.2)$ \\
\hline $1 \mathrm{yr}$ & 121 & 1 & 2 & 0 & $3(2.5)$ \\
\hline $2 \mathrm{yr}$ & 94 & 2 & 0 & 1 & $3(3.2)$ \\
\hline $3 \mathrm{yr}$ & 68 & 0 & 2 & 0 & $2(2.9)$ \\
\hline \multirow[t]{2}{*}{$4 \mathrm{yr}$} & 72 & 1 & 0 & 0 & $1(1.4)$ \\
\hline & 543 & 6 & 4 & 2 & $12(2.2)$ \\
\hline \multicolumn{6}{|c|}{$\begin{array}{l}\text { Healthy siblings of } \\
\text { patients with HIB } \\
\text { meningitis of epiglottitis }\end{array}$} \\
\hline Birth-4 yr & 29 & 1 & 4 & 14 & $19(65)$ \\
\hline
\end{tabular}

antibodies to HIB and intensity and duration of HIB colonization will be the subject of a future report.

\section{DISCUSSION}

Problems associated with detection of $H$. influenzae in the pharynx have led to the development of several media which enhance the growth of these fastidious organisms. Early methods (1918-1920) employed heating or chemical treatment of blood to provide adequate concentration of $\mathrm{X}$ and $\mathrm{V}$ factors (5-7). A transparent medium (Levinthal) provided some selectivity in that encapsulated (iridescent) strains of $H$. influenzae could be differentiated from nonencapsulated strains (7). The usefulness of inhibiting other pharyngeal organisms was recognized at about this same time with the development of a medium encorporating sodium oleate (1), which also appeared to enhance the growth of $H$. influenzae. Crystal violet (8), brilliant green (6), penicillin (4), and bacitracin (3) have been utilized to inhibit other bacteria. Recently, saponin disks have been employed on sheep blood agar plates to hemolyze erythrocytes producing a cleared zone rich in $\mathrm{V}$ factor allowing growth and observation of iridescent encapsulated $H$. influenzae (3). In spite of these developments, however, the differentiation of type $b$ from the other five capsular type strains requires additional procedures.

The principles of immunodiffusion were first applied to bacteriology by Petrie in 1932 (11), who utilized bacterial antiserum incorporated into agar to distinguish rough and smooth strains of meningococcus, pneumococcus, and $B$.dysenteriae by the halos of specific precipitates that surrounded colonies. Pittman et al. (12) used the technique to demonstrate a quantitative relation between specific precipitins and protective activity of the antisera. Ouchterlony (10) employed a similar method to quantitate the toxin-producing activity of Corynebacterium diphtheriae.

The chief advantages of this method are its rapidity and accuracy. Antiserum agar allows for specific identification of HIB in one step. Cross-reacting E. coli apparently occur rarely if at all in the pharynx of healthy children. There is no need to subculture on rabbit blood to eliminate $H$. hemolyticus, to utilize factor $\mathrm{X}$ and $V$ strips to exclude $H$. parainfluenzae, or to employ slide agglutination or the quellung reaction to eliminate nonencapsulated or other types of $H$. influenzae. The bacitracin increases sensitivity by inhibiting competing bacteria. The radial streaking with a calibrated loop provides further sensitivity and, at the same time, makes a degree of quantitation feasible. Although simple in application, the method is expensive since it is dependent on large amounts of potent and highly specific antisera, the production of a concentrated Levinthal base, and a refined agar to provide a high degree of clarity and strength.

The use of antiserum agar appears to be ideal for studies of the 
carrier rate of HIB and other encapsulated organisms including pneumococci, meningococci, and $E$. coli, since it is sensitive, highly specific, and allows rapid testing of many specimens. The results of our HIB pharyngeal carrier study also demonstrate the potential of this medium for semiquantitative studies. Other applications include studies of the role of HIB in such conditions as pneumonia, sinusitis, conjunctivitis, and orbital cellulitis of childhood.

\section{SUMMARY}

An antiserum agar medium was found to provide a rapid, sensitive, and highly specific method for pharyngeal culture and quantitation of HIB.

\section{REFERENCES AND NOTES}

1. Avery, O. T.: A selective medium for B. influenzae: Oleate hemoglobin agar. J. Amer Med Ass, 71: 2050 (1918).

2. Bradshaw, M. W., Parke, J. C., Jr., Schneerson, R., and Robbins, J. B.: Bacterial antigens cross-reactive with the capsular polysaccharide of Haemophilus influenzae type b. Lancet, $i$ : 1095 (1971).

3. Controni, G., Khan, W., Patrick, J., and Ross, S.: New techniques for the isolation and rapid identification of $H$. influenzae. Amer. J. Clin. Pathol., 49: 744 (1968).

4. Dawson, B., and Zinneman, K.: Incidence and type distribution of capsulated $H$. infleunzae strains. Brit. Med. J., i: 740 (1952).

5. Fildes, P.: A new medium for the growth of B. infleunzae. Brit. J. Exp. Pathol., I: 129 (1920).

6. Fleming. A.: On some simple prepared culture media for $B$. influenzae. Lancet. $i$ : 138 (1919).

7. Levinthal, W.: Bakteriologische und serologische influenza studien. Z. Hyg Infektionskr., 86: 1 (1918).

8. Masters, P. L., Brumfitt, W., Mendez, R. L., and Likar, M.: Bacterial flora of

Copyright ( 1975 International Pediatric Research Foundation, Inc. the upper respiratory tract in Paddington families, 1952-54. Brit. Med. J., 1: 1200 (1958).

9. Moxon, E. R., Smith, A. L., Averill, D. R., and Smith, D. H.: Haemophilus influenzae meningitis in infant rats after intranasal inoculation. J. Infect. Dis., 129: 154 (1974).

10. Ouchterlony, O.: Antigen-antibody reactions in gels. Acta Pathol. Microbiol. Scand., 26: 507 (1949).

11. Petrie, G. F.: The specific precipitin reaction associated with the growth on agar plates of meningococcus, pneumococcus, and B. dysenteriae (Shiga). Brit. J. Exp. Pathol., 13: 380 (1932).

12. Pittman, M., Branham, S. E., and Sockrider, E. M.: A comparison of the precipitation reaction in immune serum agar plates with the protection of mice by anti-meningococcus serum. Pub. Health Rep., 53: 1400 (1938).

13. Schneerson, R., Bradshaw, M., Whisnant, J. K., Myerowitz, R. L., Parke, J. C., Jr., and Robbins, J. B.: An Escherichia coli antigen cross-reactive with capsular polysaccharide of Haemophilus influenzae type b: Occurrence among known serotypes, and immunochemical and biologic properties of $E$. coli antisera to $H$. influenzae type b. J. Immunol., 108: 155! (1972).

14. Sell, S. H. W. The clinical importance of Haemophilus influenzae infections in children. Pediat. Clin. North Amer., 17: 415 (1970).

15. Shibley, G. S., Hanger, F. M., and Dochez, A. R.: Studies on the common cold. I. Observations of the normal bacterial flora of nose and throat with variations occurring during colds. J. Exp. Med., 43: 415 (1926).

16. Turk, D. C., and May, J. R. (Editors): Haemophilus influenzae: Its Clinica! Importance (The English Universities Press, Ltd., London, 1967).

17. Sigma Chemical Co., St. Louis, Mo.

18. The Upjohn Co., Kalamazoo, Mich.

19. Difco Laboratories, Detroit, Mich.

20. Marine Colloids Corp., Rockland, Me.

21. Ivan Sorvall Inc., Newtown, Conn.

22. Hyland Labs, Costa Mesa, Calif.

23. This research was supported by The Health Research and Services Foundation, Pittsburgh, $\mathrm{Pa}$

24. Requests for reprints should be addressed to: R. H. Michaels, M.D., Children's Hospital of Pittsburgh, Pittsburgh, Pa. 15213 (USA).

25. Accepted for publication January 31, 1975.

Printed in U.S.A

Pediat. Res. 9: 516 (1975)

\title{
Letter to the Editor
}

\author{
D. C. PEAKMAN, M. F. MORETON, AND A. ROBINSON
}

Cytogenetics Laboratory, Department of Biophysics and Genetics, No. 617. University of Colorado Medical Center. Denver. Colorado, USA

The report by Dr. Cox et al. (1) of chromosomal mosaicism in amniotic fluid cell cultures prompts us to report our experience with similar specimens.

In a series of 350 cultures of amniotic fluid cells, 5 showed the presence of cells with abnormal chromosome complements. Four of these demonstrated trisomy 2, the fifth involved a possible isochromosome D. In each case the abnormal cells were restricted to a single colony.
We agree with the necessity of establishing the karyotype by analyzing cells from discrete colonies in preparations processed in situ. This procedure will also make possible the identification of maternal cells which might contaminate the culture.

\section{REFERENCE}

I. Cox, D. M., Niewczas-Late, V., Riffell, M. 1., and Hamerton, J. L.: Chromosomal mosaicism in diagnostic amniotic fluid cell cultures. Pediat. Res., 8: 679 (1974). 